\title{
SARS-CoV-2 (COVID-19) as a Predictor of Neuroinflammation and Neurodegeneration: Potential Treatment Strategies
}

\author{
M. V. Putilina ${ }^{1}$ and D. V. Grishin ${ }^{1,2}$
}

Translated from Zhurnal Nevrologii i Psikhiatrii imeni S. S. Korsakova, Vol. 120, No. 8, Iss. 2, Stroke, pp. 58-64, August, 2020. Original article submitted September 11, 2020. Accepted September 14, 2020.

The SARS-CoV-2 (COVID-19) pandemic has attracted attention to the challenge of neuroinflammation as an unavoidable component of viral infections. Acute neuroinflammatory responses include activation of resident tissue macrophages in the CNS followed by release of a variety of cytokines and chemokines associated with activation of oxidative stress and delayed neuron damage. This makes the search for treatments with indirect anti-inflammatory properties relevant. From this point of view, attention is focused on further study of the treatment of patients with COVID-19 with dipyridamole (Curantil) which, having antiviral and anti-inflammatory effects, can inhibit acute inflammatory activity and progression of fibrosis, is a drug with potential, especially among patients with early increases in the D-dimer concentration and severe signs of microangiopathy.

Keywords: SARS-CoV-2 coronavirus, neuroinflammation, cytokine storm, neurodegeneration, cognitive impairment, dipyridamole.

SARS-CoV-2 coronavirus, discovered in 2019, in contrast to other known coronaviruses inducing atypical pneumonia (SARS-CoV, MERS-CoV), is more contagious and has a greater population transmission rate [1]. The course of the infection it causes, COVID-19, involves more severe clinical manifestations than those seen in acute respiratory infections and influenza. Lesions to human organs in COVID-19 are multisystem in nature, involving not only the respiratory system, but also other body systems, including the nervous system [2]. The pandemic caused by SARS-CoV-2 has attracted intense attention to neuroinflammation processes as an unavoidable component of viral infection, manifest as synaptic dysfunction, changes in intercellular interactions, degradation of macromolecules, impairments to metabolism, and, as a result, neurodegeneration. Neuroinflammation in patients with any pathology is associated with age-dependent increases in the sensitization of the immune system to external and internal stimuli [3]. Processes mediated by neuroinflammation lead to

\footnotetext{
${ }^{1}$ Pirogov Russian National Research Medical University, Russian Ministry of Health, Moscow, Russia; e-mail: profput@mail.ru.

${ }^{2}$ Filatov City Clinical Hospital No. 15, Moscow Health Department, Moscow, Russia.
}

neuropsychiatric disorders such as depression and dementia $[4,5]$. Thus, there is value in studying potential therapeutic strategies directed at preventing the development and progression of neurodegeneration, especially in elderly COVID-19 patients.

Relationship between Neuroinflammation and Neurodegeneration. Neuroinflammation is a multilevel molecular-cellular mechanisms which at the first stage supports the compensatory-adaptive responses of the brain, subsequently activating neurodegenerative processes [6]; it is associated with activation of proinflammatory cytokines in response to contact with pathogenic factors (metabolic, toxic, infectious, and traumatic, including chronic stress). Acute neuroinflammatory responses include activation of resident tissue macrophages in the central nervous system (CNS) and subsequent release of various cytokines and chemokines, activating oxidative stress and inducing long-term neuron damage and the development of coagulatory cascades inducing endothelial cell damage [7]. Inflammatory cytokines are a type of signal molecule released from immune cells such as T-helpers (Th) and macrophages. These include not only interleukin (IL)-6, but also IL-1 $,-8,-12$, and -18 , tumor necrosis factor $\alpha$ (TNF- $\alpha$ ), and $\gamma$-interferon (IFN- $\gamma$ ). 
Endothelial cells are a key component in the bloodbrain barrier (BBB), so endothelial dysfunction is always associated with damage to the BBB. Impairments to the integrity of the BBB allow various types of cells to enter the damaged part of the brain [8]. Migrating immunocompetent cells (along with damaged cells) produce inflammatory mediators, accelerating neuron death [9]. Endogenous danger-associated molecular pattern (DAMP) molecules are released from the intracellular space of dead or damaged cells, and these are believed to be activators of the microglia and brain tissue-infiltrating peripheral immunocompetent cells [10-14]. Microglia activated by high-mobility group box 1 (HMGB1) secrete TNF- $\alpha$, IL-1 $\beta$, reactive oxygen species (ROS), and numerous proinflammatory cytokines $[11,12]$, which initiate inflammatory reactions in the brain in response to harmful stimuli $[13,14]$. Increases in the number of activated glial cells and the concentrations of a number of cytokines (such as TNF- $\alpha$ ) in the hippocampus, cerebral cortex, substantia nigra, and striatum are associated with decreases in hippocampal volume and cognitive dysfunction due to the neurodegenerative process [15].

Apart from microglia, damaged astrocytes and pericytes can produce proinflammatory cytokines [15]. Activated astrocytes form a glial scar, limiting the potential for recovery of damaged axons. Pericytes located along capillary walls provide density to the BBB. Damage (hypoxia, ischemia, infection) impairs BBB density, leading to impairment of intercellular circulation in the perivascular spaces with activation of macrophages via development of local inflammation [16]. Apart from the local immune response, damage to neurovascular units can produce the phenomenon of systemic suppression of immunity, which is clinically apparent as increases in susceptibility to viral and bacterial infections, including pneumonia or lower urinary tract infections $[17,18]$. Cytokine reactions make a significant contribution to the mechanism of CNS damage leading to neuron degeneration in Alzheimer's disease, Parkinson's disease, stroke, etc. [4].

The Role of Neuroinflammation in the Pathogenesis of COVID-19. Thus far, the pathogenesis of damage associated with SARS-CoV-2 has received insufficient study. SARS-CoV-2 is known to have a genome sequence close to that of SARS-CoV-1 [1]. Both viruses use protein spikes on their surfaces to bind the angiotensin converting enzyme 2 (ACE-2) receptor in mammalian cells, after which membrane-bound serine protease 2 activates the spikes [19]. Unlike other viruses of this group, the rate of entry of SARSCoV-2 into cells is linked with preliminary preactivation of the peplomer (spike) by furin. After infection, the virus spreads through the respiratory tract, inducing release of proinflammatory cytokines. A feature of this infection is the presence of extramedullary megakaryocytes which actively produce platelets [20]. There is a simultaneous reduction in the blood lymphocyte count, particularly T-lymphocytes, and suppression of endogenous immune system mechanism with development of secondary hemophagocyte lymphohis- tiocytosis [21]. The term cytokine storm (CS) was applied to these processes, referring to uncontrolled inflammation with elevated synthesis of numerous inflammation markers (C-reactive protein, IL-6, etc.), leading to multiorgan failure and CNS damage [1]. A CS is initiated by interaction of spike proteins and other protein particles on the coronavirus capsid with cell surface receptors. The interaction of viruses with toll-like receptors triggers the NF- $x \mathrm{~B}$ inflammatory signal cascade. Activation of NF- $x \mathrm{~B}$ stimulates prointerleukin-1 secretion, and this undergoes proteolysis by caspase-1, leading to activation of inflammasomes and synthesis of active IL- $1 \beta$. In turn, IL-1 $\beta$, stimulating the secretion of other proinflammatory cytokines, provokes the development of pulmonary fibrosis and fever [21, 22]. Increased proinflammatory cytokine secretion and imbalance in the expression of the corresponding receptors and their associated proteins (the main component of the "SARS-CoV-2 phenomenon") result from the ineffective realization of the anti-inflammatory response with damage to various organs $[3,4]$.

In the CNS, the immune response is produced by cells of endogenous origin - microglia and endothelial cells, as well as exogenous cells, i.e., dendritic cells, T- and B-lymphocytes, and cells of the mononuclear phagocyte system. Entry of virus into the CNS occurs immediately after infection. Evidence has been obtained showing similarity between CNS lesions in COVID-19 and those induced by herpes simplex virus and HIV, as all infect neurons via fast retrograde axonal transport [19]. SARS-CoV-2 is simultaneously neurotropic and neurovirulent. It is able to penetrate into the CNS by neuronal dissemination, in which the virus initially infects peripheral neurons, and then, using the host cell apparatus, infects CNS neurons, inducing a set of consequences related to the disease, including neurodegenerative. This mechanism of propagation is termed the "Trojan horse" mechanism [23]. The CoV spike glycoprotein used by SARS-CoV-2 to bind to cell membranes induces ACE2 receptor expression in neurons and endothelial cells and determines the neuroinvasive potential of the virus. Cells bearing CD4 receptors become targets of the virus - macrophages, neuroglia, and capillary endothelia. After crossing the BBB, the virus can replicate in microglia and neurons, producing corresponding damage to the BBB. Infected microglial cells produce low molecular weight peptides with toxic actions on astrocytes, leading to excessive accumulation of glutamate in the extracellular space and development of glutamate excitotoxicity. These processes occur on the background of impairments to brain blood flow autoregulation, vasospasm, increased platelet aggregation, and formation of intravascular stasis, impaired microcirculation, and endothelial dysfunction.

Impairments to endothelial function result in sharp changes in the spectrum of active substances produced by them [7]. The endothelium starts to secrete aggregants, coagulants, and vasoconstrictors, some of which (the renin-angiotensin system) affect the whole cardiovascular 
system. Increases in blood coagulability are linked with a high risk of death from COVID-19 [23]. Increased blood plasmin and plasminogen concentrations are biomarkers for increased susceptibility to SARS-CoV-2, as plasmin protease can cleave the corresponding site on SARS-CoV-2 S protein, increasing its virulence [24].

The precise mechanism of development of coagulopathy and microangiopathy in COVID-19 is unknown. This is probably linked with diffuse inflammation or damage to endothelial cells [25]. The most widespread damage liked with thrombotic microangiopathy are thrombotic thrombocytopenic purpura and hemolytic uremia syndrome. Patients probably develop so-called small vessel disease. This term is used to describe a series of syndromes, whose pathogenesis is quite unclear and may be linked with damage to the perforant cerebral arterioles, capillaries, and venules [26]. The clinical signs of small vessel disease are beyond the framework of clear acute syndromes and can be apparent as changes in mood and depression, balance problems, falls in the elderly, reversible amnesia, and autonomic dysfunction [5]; furthermore, small vessel disease itself is the commonest cause of silent strokes.

The most vulnerable patients in COVID-19 are the elderly, with comorbid states such as heart disease, kidney disease, arterial hypertension, diabetes mellitus, obesity, and atherosclerosis [27-29]. Development of viral infection in these patients is associated with increased levels of markers for liver dysfunction (aspartate aminotransferase, alanine aminotransferase, albumin, bilirubin), along with severe impairments to coagulation. As a rule, these findings are prognostically unfavorable indicators and provide evidence of a more severe course of pneumonia and progression of gastrointestinal tract damage [30]. The presence of chronic comorbid diseases in these patients explains the more severe course of COVID-19 in the elderly. A multicenter Chinese trial $(n=280)$ showed that severe disease was more commonly seen in patients more than 65 years old, $85.5 \%$ of patients with severe diseasing having diabetes mellitus, arterial hypertension, or angina, which were encountered at frequencies $7-10$ times greater $(p=0.042)$ than in patients with mild COVID-19 [30]. Decompensation of any somatic or infectious disease has significant influences on the state of cognitive functions in elderly patients because of the large number of pathogenic factors.

Patients with other diseases and new virus infection are in a "vicious circle": on the one hand, arterial hypertension, especially when poorly controlled, and diabetes mellitus are known to be directly linked with small vessel damage in the brain and cognitive changes [31]. On the other hand, patients with impaired cognitive functions are at elevated risk of COVID-19 infection, as they are often unable to obtain or understand the required information on the importance of the problem and methods of preventing disease. Such patients are not aware of the need for prophylaxis of disease or strict observation of self-isolation regimes. Their limited access to current information (especially when living alone) can have the result that the early symptoms of the disease will not be recognized, leading to the risk of progression of infection and the development of complications [32]. The early neurological manifestations of COVID-19 can be general weakness, apathy, loss of appetite, asthenia, and perhaps episodic memory loss at the initial stages, with difficulty committing information to memory. The later stages can be linked with total amnesia, progression of cognitive impairments, disorientation, confusion, hallucinations, delusions, and depression [33, 34]. Severe depression can lead to impairments to cognitive functions and can even simulate dementia.

The neurological signs of COVID-19 usually appear immediately after infection, though they can also develop at later stages of the disease or after recovery [27]. Patients with COVID-19 have also been found to develop neurological symptoms such as headache, vertigo, myalgia, and anosmia, and cases of encephalitis, necrotizing hemorrhagic encephalopathy, stroke, epileptic seizures, delirium, rhabdomyolysis, and Guillain-Barré syndrome have also been recorded [28, 29].

Asthenic disorders are dominant among patients with virus infection. The most frequent are oscillations in arterial blood pressure, tachycardia, and pulse rate lability, a diversity of pains or simply unpleasant sensations, erythema or pallor of the skin, feelings of heat at normal body temperature, or, conversely, elevated chilliness, and local (palms, feet, armpits) or generalized hyperhidrosis. Patients rarely complain of melancholy or low mood; more typical is painful fixation on unpleasant somatic sensations, which cannot be fully explained in terms of the ongoing diseases. Typical complaints are headache, pain in the back, joints, and internal organs, vertigo, and noises and sounds in the head [34, 35].

Possible Therapeutic Strategies in COVID-19: A Focus on Neuroinflammation. The current therapeutic strategy for the treatment of neurodegenerative diseases is based mainly on correction of clinical symptoms, though its efficacy in some patients is minimal, as it does not always take account of the complex pathogenetic mechanisms, including neuroinflammation, etiological factors, and the features of the clinical picture. Selection of medication requires consideration of the fact that cognitive impairments in patients with COVIS-19 may be a manifestation not of the direct neurodegenerative process, but can be indirectly associated with the development of hypoxic encephalopathy [36]. Impairments to neurotransmitter functions (insufficient or, conversely, excessive reactivity) causes disorganization of synaptic processes and is always synergistic with hypoxia in ischemia or virus infection, so selection of first-line drugs is always difficult.

In the current absence of guideline documents for the treatment of COVID-19, it is difficult to say whether any strategy has advantages, so drugs fitting with the majority of therapeutic approaches should be chosen. Primary is the need to correct comorbid states [37]. 
Antihypertensive drugs and statins are medications whose influences on the functional state of the endothelium have received the most study $[38,39]$. There are as yet no experimental or clinical data evidencing favorable or unfavorable influences of treatment with angiotensin converting enzyme inhibitors, renin-angiotensin-aldosterone system blockers, and other antihypertensive drugs on the prognosis and course of illness in patients with COVID-19 or patients with COVID-19 with cardiovascular disease and receiving treatment with these drugs.

The role of systemic inflammation provides grounds for using anti-inflammatory drugs. At the same time, the incidence of side effects when these are used in the long term (hypertension, hyperglycemia, osteoporosis, increased risk of cardiovascular events, gastrointestinal tract pathology) makes their prescription inadvisable. Many other drugs also have anti-inflammatory actions, with reductions in the activity of the cellular components of inflammation (activation of neutrophils and monocytes, as well as leukocyte-platelet conjugates) and/or soluble biomarkers (such as C-reactive protein, cytokines, and interleukins). A series of pilot studies with drugs inhibiting IL-1 and IL- $6 \beta$ have shown that their use in addition to standard treatment improves disease outcomes, though they have a number of contraindications; in particular, there is a lack of data on influences on cognitive functions, especially in the long-term phase of COVID-19 [40].

As a result of the decrease in the arachidonic acid lev$\mathrm{el}$, the signs of CS, and other factors, the use of antiplatelet drugs is currently regarded as having potential in the treatment of COVID-19 [41-43]. These can increase the level of adaptation of neurons to damage, increasing their viability in unfavorable conditions and thus providing optimum restoration of blood flow in damaged vessels [44]. Recent years have seen many reports confirming the role of platelets as key signal and effector cells in overcoming the hemostatic, inflammatory, and immune continuums in infectious and viral diseases [45]. These cells, binding virus via Fc receptors or plasma proteins, activate the release of "microbial" proteins and peptides by platelets, including platelet factor PF-4, T-cells, recombinant human protein (RANTES), and fibrinopeptide B. RANTES operates as a chemoattractant for blood monocytes, T-helpers, and eosinophils; the protein induces histamine release from basophils and activates eosinophils. Acting via a diverse set of mechanisms, activated platelets can isolate and kill pathogens or promote their elimination from the body via macrophage and neutrophil activation, enhancing the generation of neutrophil extracellular traps and the formation of platelet aggregates and microthrombi.

Published clinical studies run in China have shown that the antiplatelet drug dipyridamole has anti-HCoV-19 effects [44]. In a model of viral pneumonia, it activated antiviral immunity and significantly improved survival, displaying marked antiaggregant actions. In clinical practice, addition- al treatment with dipyridamole led to increases in the numbers of circulating lymphocytes and platelets, decreases in the D-dimer level, and a notable improvement in clinical outcomes. Dipyridamole inhibits the expression of proinflammatory cytokines (IL-1, -2) and TNF- $\alpha$, largely slowing the translocation of the p65 subunit of nuclear factor $x \mathrm{~B}$ $\mathrm{NF}-x \mathrm{~B}$, which increases the activation, differentiation, and effector functions of T-cells and inflammasomes [46-48]. Dipyridamole has systemic and local anti-inflammatory effects, significantly reducing the level of matrix metalloproteinase-9 in monocytes [48]. As a pyrimidine derivative, dipyridamole is an interferon inducer and has a modulatory action on the functional activity of the interferon system; it reverses decreased IFN- $\alpha$ and IFN- $\gamma$ production by in vitro blood leukocytes; it increases nonspecific antiviral resistance to viral infections [47]. The drug has powerful antiviral effects in relation to single-stranded RNA viruses in vitro and in an in vivo model of VSV-induced viral pneumonia; it displays an interferon-modulating action in recurrent opportunistic viral infections on the background of chronic emotional stress.

Dipyridamole suppresses SARS-CoV-2 replication, suggesting that the therapeutic dose of the drug may potentiate the antiviral reactions of infected patients [44]. Furthermore, as a phosphodiesterase inhibitor, dipyridamole has additional advantages in patients with new viral infections, as it has a vasodilating action, increasing the adenosine level, inhibits phosphodiesterase in vascular smooth muscle cells, and is a classical endothelioprotector [49]. In addition to the antithrombotic and vasodilating effects, the antioxidant and endothelioprotector actions of dipyridamole may slow neurodegenerative processes, improving cognitive functions [50].

Excessive quantities of proinflammatory cytokines, especially IL-1, lead to expression of brain-derived neurotrophic factor [40], so restoration of neurotrophics is directly associated with the extent of reductions in the severity of CS. A potential direction for treatment is provided by use of neurotrophic drugs [51].

Conclusions. The medical community continues to lack full information on the pathogenic actions of COVID-19 virus and its possible consequences for the nervous system. However, existing data lead to the view that the virus activates inflammation processes and neurodegeneration with subsequent manifestation of cognitive impairments. This confirms the need for seeking drugs acting on the whole neurovascular unit with indirect or direct anti-inflammatory effects with demonstrated efficacy. From this point of view, further study of the treatment of COVID-19 patients with dipyridamole, whose antiviral and anti-inflammatory actions inhibit acute inflammation and the progression of fibrosis, is a potentially valuable drug, especially in patients with the early signs of increased D-dimer concentrations and signs of microangiopathy.

The authors have no conflicts of interests. 


\section{REFERENCES}

1. X. Jin, J. S. Lian, J. H. Hu, et al., "Epidemiological, clinical and virological characteristics of 74 cases of coronavirus-infected disease 2019 (COVID-19) with gastrointestinal symptoms," Gut (2020), pii: Gutjnl-2020-320926, https://doi.org/10.1136/gutjnl-2020-320926.

2. J. Wu, W. Li, X. Shi, et al., "Early antiviral treatment contributes to alleviate the severity and improve the prognosis of patients with novel coronavirus disease (COVID-19)," J. Intern. Med. (2020), https://doi.org/10.1111/joim.13063.

3. S. Amor, F. Puentes, D. Baker, and P. van der Valk, "Inflammation in neurodegenerative diseases," Immunology, 129, No. 2, 154-169 (2010), https://doi.org/10.1111/j.1365-2567.2009.03225.x.

4. W. J. Streit, Q. S. Xue, H. Braak, and K. del Tredici, "Presence of severe neuroinflammation does not intensify neurofibrillary degeneration in human brain," Glia, 62, No. 1, 96-105 (2014), https://doi. org/10.1002/glia.22589.

5. A. K. Walker, A. Kavelaars, C. J. Heijnen, and R. Dantzer, "Neuroinflammation and comorbidity of pain and depression," Pharmacol. Rev., 66, No. 1,80-101 (2013), https://doi.org/10.1124/pr.113.008144.

6. G. Singhal, E. J. Jaehne, F. Corrigan, et al., "Inflammasomes in neuroinflammation and changes in brain function: a focused review," Front. Neurosci., 8, 315 (2014), https://doi.org/10.3389/fnins.2014.00315.

7. M. V. Putilina, "The endothelium as a target for new therapeutic strategies in cerebral vascular diseases," Zh. Nevrol.Psikhiatr., 117, No. 10, 122-130 (2017), https://doi.org/10.17116/jnevro2017117101122-130.

8. E. Gülke, M. Gelderblom, and T. Magnus, "Danger signals in stroke and their role on microglia activation after ischemia," Ther. Adv. Neurol. Disord., 22, No. 11, 1756286418774254 (2018), https://doi. org/10.1177/1756286418774254.

9. Á. Chamorro, U. Dirnagl, X. Urra, and A. M. Planas, "Neuroprotection in acute stroke: targeting excitotoxicity, oxidative and nitrosative stress, and inflammation," Lancet Neurol., 15, No. 8, 869-881 (2016), https://doi.org/10.1016/S1474-4422(16)00114-9.

10. G. Y. Chen and G. Nuñez, "Sterile inflammation: sensing and reacting to damage," Nat. Rev. Immunol., 10, No. 12, 826-837 (2010), https://doi.org/10.1038/nri2873.

11. M. G. Frank, M. D. Weber, L. R. Watkins, and S. F. Maier, "Stress sounds the alarmin: The role of the danger-associated molecular pattern HMGB1 in stress-induced neuroinflammatory priming," Brain Behav. Immun., 48, 1-7 (2015), https://doi.org/10.1016/j.bbi.2015. 03.010.

12. A. Fettelschoss, M. Kistowska, S. Leibund Gut-Landmann, et al., "Inflammasome activation and IL-1 $\beta$ target IL- $1 \alpha$ for secretion as opposed to surface expression," Proc. Natl. Acad. Sci. USA, 108, No. 44, 18055-18060 (2011), https://doi.org/10.1073/pnas.1109176108.

13. O. V. Artem'eva and L. V. Gankovskaya, "Inflammatory aging as the basis of age-associated diseases," Med. Immunol. (Russia), 3, No. 22, 419-432 (2020), https://doi.org/10.15789/1563-0625-IAT-1938.

14. B. Lima Giacobbo, J. Doorduin, H. C. Klein, et al., "Brain-derived neurotrophic factor in brain disorders: Focus on neuroinflammation," Mol. Neurobiol., 56, No. 5, 3295-3312 (2019), https://doi.org/ 10.1007/s12035-018-1283-6.

15. A. I. Chernykh, Yu. K. Komleva, Ya. V. Gorina, et al., "Proinflammatory phenotype of perivascular astroglia and $\mathrm{CD} 133+$ progenitor cells of endotheliocytes in A murine model of Alzheimer's disease," Fundament. Klin. Med., 3, No. 1, 6-15 (2018), https://doi.org/10. 23946/2500-0764-2018-3-1-6-15.

16. V. S. S. S. Sajja, N. Hlavac, and P. J. VandeVord, "Role of glia in memory deficits following traumatic brain injury: Biomarkers of glia dysfunction.," Front. Integr. Neurosci. (2016), https://doi.org/ 10.3389/fnint.2016.00007.

17. S. Y. Liang, "Sepsis and other infectious disease emergencies in the elderly," Emerg. Med. Clin. North Am., 34, No. 3, 501-522 (2016), https://doi.org/10.1016/j.emc.2016.04.005.
18. G. Zhang, J. Li, S. Purkayastha, et al., "Hypothalamic programming of systemic ageing involving IKK- $\beta, \mathrm{NF}-\varkappa \mathrm{B}$ and GnRH," Nature, 497, No. 7448, 211-216 (2013), https://doi.org/10.1038/nature12143.

19. M. Hoffmann, H. Kleine-Weber, S. Schroeder, et al., "SARS-CoV-2 cell entry depends on ACE2 and TMPRSS2 and is blocked by a clinically proven protease inhibitor," Cell, 181, No. 2, 271-280.e8 (2020), https://doi.org/10.1016/j.cell.2020.02.052.

20. A. J. Rodriguez-Morales, J. A. Cardona-Ospina, E. Gutiérrez-Ocampo, et al., "Clinical, laboratory and imaging features of COVID-19: A systematic review and meta-analysis.," Travel Med. Infect. Dis., 34, 101623 (2020), https://doi.org/10.1016/j.tmaid.2020.101623.

21. P. Conti, G. Ronconi, A. Caraffa, et al., "Induction of pro-inflammatory cytokines (IL-1 and IL-6) and lung inflammation by Coronavirus-19 (COVI-19 or SARS-CoV-2, anti-inflammatory strategies," J. Biol.Regul. Homeost. Agents, 34, No. 2 (2020), https://doi.org/10. 23812/CONTI-E.

22. L. A. Borthwick, "The IL-1 cytokine family and its role in inflammation and fibrosis in the lung," Semin. Immunopathol., 38, No. 4, 517-534 (2016), https://doi.org/10.1007/s00281-016-0559-z.

23. A. S. Zubair, L. S. McAlpine, T. Gardin, et al., "Neuropathogenesis and neurologic manifestations of the coronaviruses in the age of coronavirus disease 2019: A review," JAMA Neurol., 77, No. 8, 1018-1027 (2020), https://doi.org/10.1001/jamaneurol.2020.2065.

24. R. C. Becker, "COVID-19 update: Covid-19-associated coagulopathy," J. Thromb. Thrombolysis, 50, No. 1, 54-67 (2020), https://doi. org/10.1007/s11239-020-02134-3.

25. A. Amgalan and M. Othman, "Exploring possible mechanisms for COVID-19 induced thrombocytopenia: Unanswered questions," J. Thromb. Haemost., 18, No. 6, 1514-1516 (2020), https://doi.org/ 10.1111/jth.14832.

26. M. V. Putilina, "Current concepts of small vessel disease," $Z h$. Nevrol. Psikhiatr., 119, No. 11, 65-73 (2019), https://doi.org/10. 17116/jnevro201911911165.

27. F. J. Carod-Artal, "Neurological complications of coronavirus and COVID-19," Rev. Neurol., 70, No. 9, 311-322 (2020), https://doi. org/10.33588/rn.7009.2020179.

28. L. Mao, M. Wang, S. Chen, et al., "Neurological manifestations of hospitalized patients with COVID-19 in Wuhan, China: a retrospective case series study," JAMA Neurol., 77, No. 6, 683-690 (2020), https://doi.org/10.1001/jamaneurol.2020.1127.

29. A. Acharya, B. D. Kevadiya, H. E. Gendelman, and S. N. Byrareddy, "SARS-CoV-2 infection leads to neurological dysfunction," J. Neuroimmune Pharmacol., 15, No. 2, 167-173 (2020), https://doi.org/ 10.1007/s11481-020-09924-9.

30. W. Guan, Z. Ni, Y. Hu, et al., "Clinical characteristics of coronavirus disease 2019 in China," N. Engl. J. Med. (2020), Epub ahead of print, https://www.nejm.org/doi/full/10.1056/NEJMoa2002032.

31. V. N. Lyusov, E. M. Evsikov, and N. V. Teplova, "Etiology and factors in the development and progression of severe and malignant arterial hypertension," Ross. Kardiol. Zh., 4, No. 14, 6-16 (2009), https://doi.org/10.15829/1560-4071-2009-4-6-16.

32. P. Martin-Jimenez, M. I. Munoz-Garcia, D. Seoane, et al., "Cognitive impairment is a common comorbidity in COVID-19 deceased patients. A hospital-based retrospective cohort study," medRxiv (2020), https://doi.org/10.1101/2020.06.08.20125872.

33. A. Filatov, P. Sharma, F. Hindi, and P. S. Espinosa, "Neurological complications of coronavirus disease (COVID-19). Encephalopathy," Cureus, 12, No. 3, e7352 (2020), https://doi.org/10.7759/cureus.7352.

34. C. Wilcox and H. Zhou, "The landscape of cognitive function in recovered COVID-19 patients," J. Psychiatr. Res., 129, 98 (2020), https://doi.org/10.1016/j.jpsychires.2020.06.022.

35. A. Hall, T. Pekkala, and T. Polvikoski, "Prediction models for dementia and neuropathology in the oldest old: the Vantaa $85+$ cohort study," Alzheimers Res. Ther., 11, No. 1, 11 (2019), https://doi.org/ 10.1186/s13195-018-0450-3. 
36. M. V. Putilina and E. B. Natarova, "Features of manifestations of cerebral circulatory insufficiency in young patients," Ross. Med. Vesti, No. 1, 41-44 (2002).

37. Prevention, Diagnosis and Treatment of New Coronavirus Infection (COVID-19). Provisional Guidelines, Ministry of Health of the Russian Federation, Ver. 7, June 3, 2020.

38. M. V. Putilina and M. A. Soldatov, "Cerebral strokes in the elderly. Features of the clinical picture, course, and treatment," Vrach, $\mathbf{5}$, 29-34 (2006)

39. N. V. Teplova and E. M. Evsikov, "The angiotensin receptor blocker valsartan (Diovan) in clinical practice," Ross. Med. Zh., 14, 94-97 (2005).

40. G. Cavalli, G. De Luca, C. Campochiaro, et al., "Interleukin-1 blockade with high-dose anakinra in patients with COVID-19, acute respiratory distress syndrome, and hyperinflammation: a retrospective cohort study," Lancet Rheumatol., 2, 325-331 (2020), https:// doi.org/10.1016/S2665-9913(20)30127-2.

41. B. M. Philip and J. M. Wardlaw, "Pharmacological treatment and prevention of cerebral small vessel disease: a review of potential interventions," Int. J. Stroke, 10, No. 4, 469-478 (2015), https://doi. org/10.1111/ijs.12466.

42. M. V. Putilina, "Combined use of neuroprotectors in the treatment of cerebrovascular diseases," Zh. Nevrol. Psikhiatr., 116, No. 11, 58-63 (2016), https://doi.org/10.17116/jnevro201611611158-63.

43. L. Tingbo, Handbook of COVID-19. Prevention and Treatment (2020), https://www.zju.edu.cn/english/2020/0323/c19573a1987520/ page.htm.
44. X. Liu, Z. Li, S. Liu, et al., "Therapeutic effects of dipyridamole on COVID-19 patients with coagulation dysfunction," medRxiv, 2020. 02.27.20027557, https://doi.org/10.1101/2020.02.27.20027557.

45. L. Guo and M. T. Rondina, "The era of thromboinflammation: platelets are dynamic sensors and effector cells during infectious diseases," Front. Immunol., 10, 22-34 (2019), https://doi.org/10.3389/ fimmu.2019.02204.

46. S. Kalayoglu Besisik, M. Ozbalak, Y. B. Tor, et al., "Dipyridamole added to anticoagulant prophylaxis: decline in poor outcome of clinically severe ill COVID-2019 patients [abstract]," Res. Pract. Thromb. Haemost., 4, Suppl. 1 (2020), https://abstracts.isth.org/abstract/dipyridamole-added-to-anticoagulant-prophylaxis-decline-in-poor-outcome-of-clinically-severe-ill-covid-2019-patients/, acc. Aug. 17, 2020.

47. E. N. Kareva, "Features of the pharmacological actions and use of dipyridamole in the prevention and treatment of viral infections," Consilium Medicum, 18, No. 12, 80-87 (2016).

48. P. Balakumar, Y. Nyoa, and R. Renushiaa, "Classical and pleiotropic actions of dipyridamole: Not enough light to illuminate the dark tunnel?" Pharmacol.Res.,87,144-150 (2014), https://doi.org/10.1016/j. phrs.2014.05.008

49. M. V. Putilina, "The Role of endothelial dysfunction in cerebrovascular diseases," Vrach, 7, 24-28 (2012).

50. M. M. Tanashyan and M. A. Domashenko, "Use of Curantil in chronic cerebrovascular disease," Atmosfera. Nervnye Bolezni, 3, 8-11 (2005).

51. A. A. Yakovlev and N. V. Gulyaeva, "Molecular partners of cortexin in the brain," Neirokhimiya, 33, 1:91-96 (2017), https://doi.org/10. $1134 / \mathrm{S} 1819712416040164$ 